\title{
Synthesis and Surface Modification of Highly Monodispersed, Spherical Gold Nanoparticles of 50-200 nm
}

Steven D. Perrault, Warren C.W. Chan.

\section{Supplementary Information}

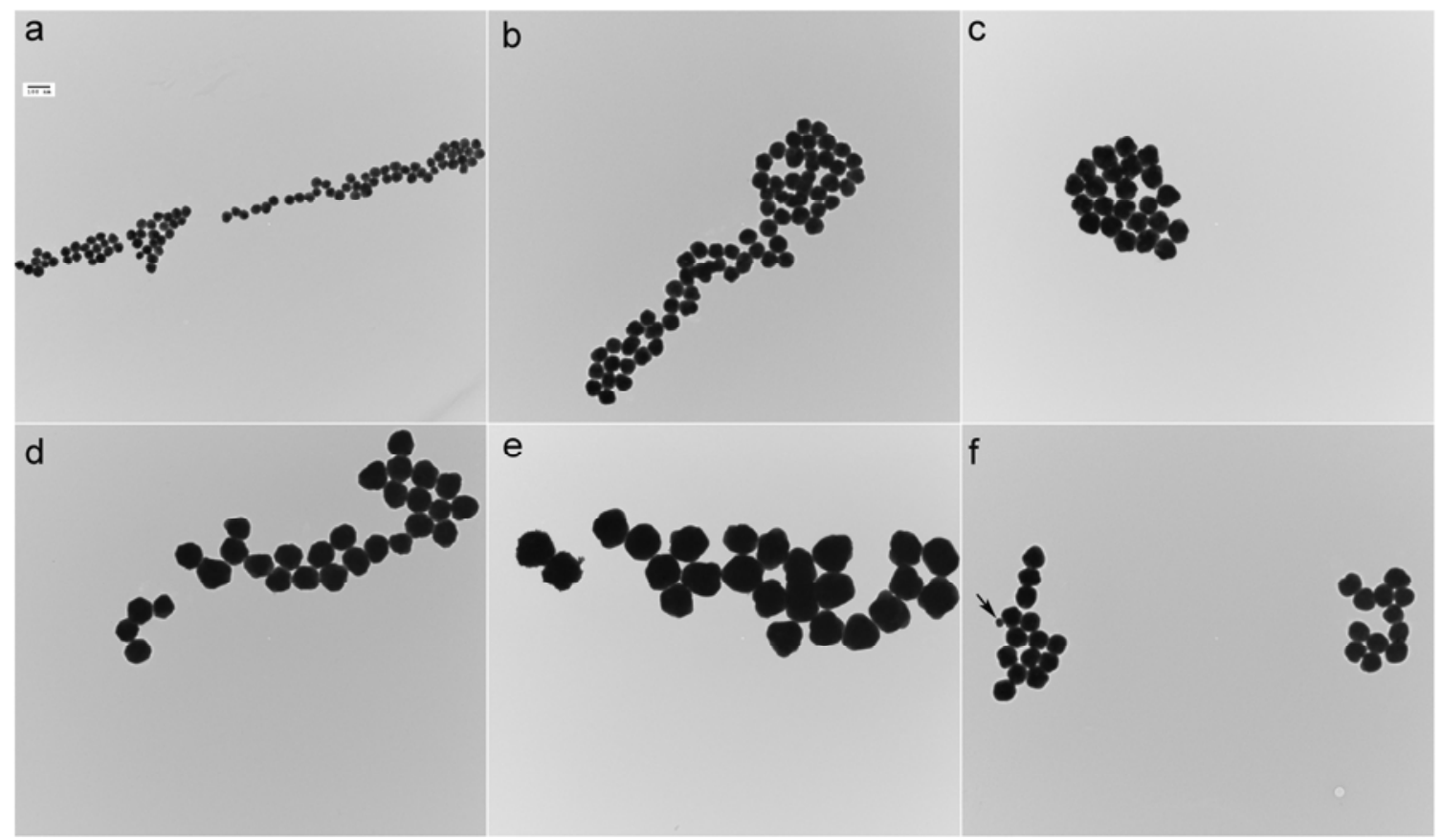

Supplementary Figure 1. Representative images of 5 particle sizes and example of new nucleation. a) $50.0 \mathrm{~nm}$, b) $74.8 \mathrm{~nm}$, c) $95.0 \mathrm{~nm}$, d) $127.3 \mathrm{~nm}$, e) $175.7 \mathrm{~nm}$ particles. f) $95.0 \mathrm{~nm}$ particles, with the arrow pointing to a small particle $(41.2 \mathrm{~nm})$ which may be an example of a newly nucleated particle from the synthesis. The scale bar in (a) of $100 \mathrm{~nm}$ is applicable to all images. 


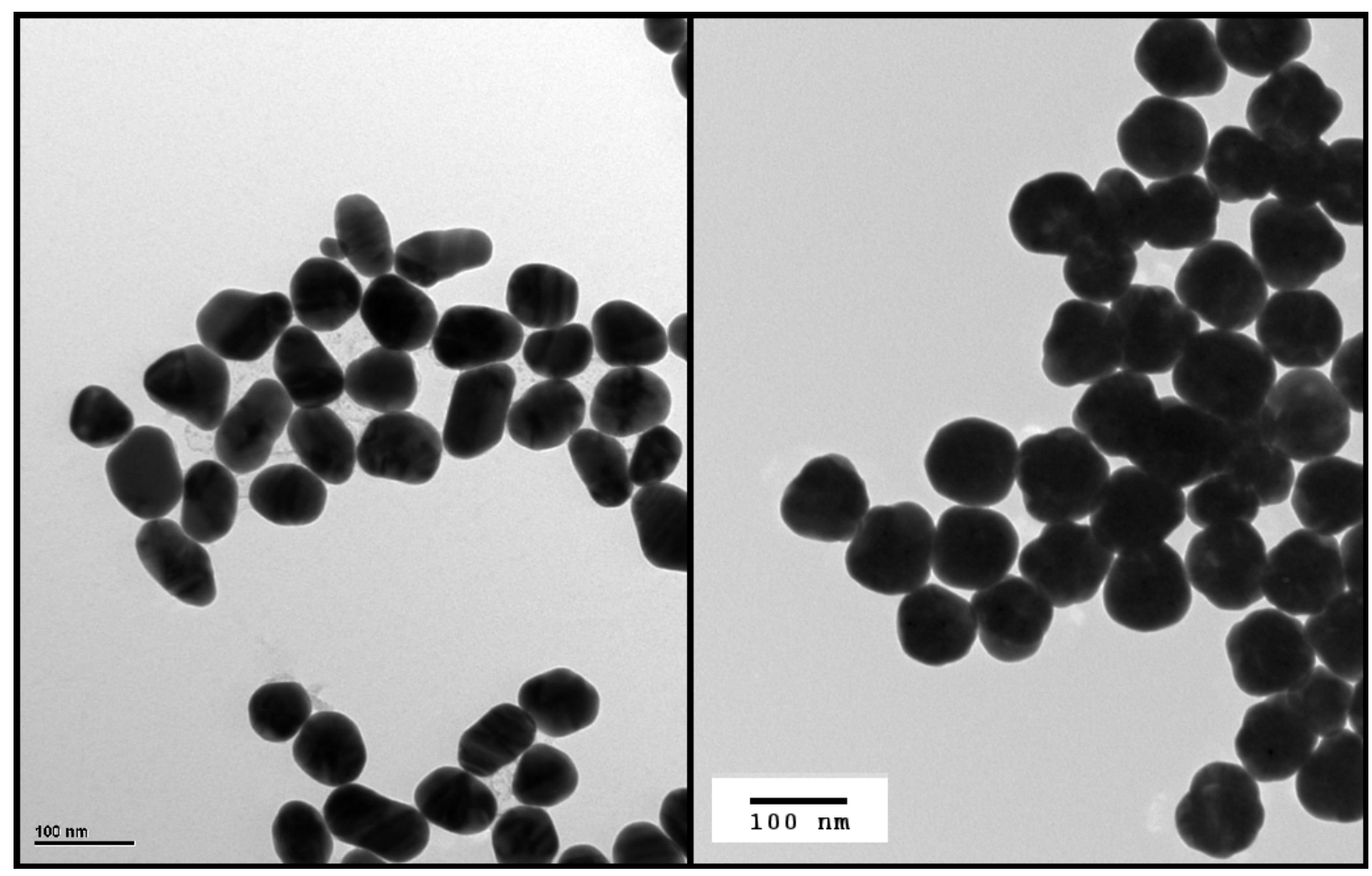

Supplementary Figure 2. Representative image of $82.51 \mathrm{~nm}$ particles synthesized by the sodium citrate reduction method (left) and $84.95 \mathrm{~nm}$ particles synthesized by the hydroquinone reduction method (right). Particles $>50 \mathrm{~nm}$ synthesized by sodium citrate typically are poorly monodispersed, and are very non-spherical, whereas the HQ method produced monodispersed, spherical particles. 


\section{Materials and Methods}

\section{Hydroquinone Synthesis of Gold Nanoparticles}

A gold nanoparticle seed solutions was prepared by sodium citrate reduction. $30 \mathrm{ml}$ of ultra-pure $\mathrm{H}_{2} \mathrm{O}$ and 300 ul of $1 \%$ (W/V) $\mathrm{HAuCl}_{3}$ (Sigma-Aldrich) was added to a $250 \mathrm{ml}$ Erlenmeyer flask with a clean stir rod. This was rapidly brought to a boil while stirring at the maximum speed possible that did not cause splashing of the solution. As soon as the solution was boiling, 900 ul of $1 \%$ (W/V) sodium citrate trihydrate (Sigma-Aldrich) solution was added. The flask was removed from heat after 10 minutes, once nanoparticle maturation was complete as indicated by colour transition. Large diameter GNP used to compare citrate reduction against $\mathrm{HQ}$ were synthesized in a similar manner, using $180 \mathrm{ul}$ of $1 \%$ sodium citrate instead of $900 \mathrm{ul}$.

Hydroquinone-reduction particles were synthesized in $10 \mathrm{ml}$ batches. Hydroquinone and sodium citrate solutions were prepared fresh each day. Gold chloride (Sigma-Aldrich) solution was centrifuged at $18,000 \mathrm{x} \mathrm{g}$ for 60 minutes in a microcentrifuge tube before use to remove any aggregates, after which volumes were obtained off the top. Sodium citrate was not included in the reactions used to generate the kinetic data (Figure 1a), but was otherwise included. For a given synthesis, 100 ul of a $1 \%(\mathrm{~W} / \mathrm{V}) \mathrm{HAuCl}_{3}$ solution was added to $9.4-9.8 \mathrm{ml}$ of ultra-pure $\mathrm{H}_{2} \mathrm{O}$ (depending on balance of water added with seeds) in a $20 \mathrm{ml}$ scintillation vial. The appropriate volume of particle seeds was then added (minimum of $7.7 \mathrm{ul}$ and maximum of $406 \mathrm{ul}$ ). The solution was then stirred rapidly at room temperature. $22 \mathrm{ul}$ of a $1 \%$ sodium citrate solution was then added, immediately followed by $100 \mathrm{ul}$ of $0.03 \mathrm{M}$ hydroquinone (Sigma-Aldrich). Reduction was immediately apparent in reactions containing a high ratio of seeds, and completed within approximately 10 minutes, whereas reactions with low ratios of seeds took up to 60 minutes to complete.

\section{Particle Pegylation}

Concentration of the $50 \mathrm{~nm}$ particles was determined first, using Beer's Law and the following equation to determine the particle's extinction coefficient:

$$
10^{\left(1.0643^{*} \log \left(3 / 2^{*} 3.141592654^{*}(\text { diameter })\right)+4.0935\right)}
$$

Based on the concentration and particle size, we estimated the available surface area $\left(\mathrm{nm}^{2} / \mathrm{ml}\right)$. We then prepared pegylation reactions. A 1.0 and $0.1 \mathrm{mg} / \mathrm{ml}$ solution of $\mathrm{mPEG}-\mathrm{SH}$ (M.W. $=5000$, Laysan Bio.) was prepared in ultra-pure $\mathrm{H}_{2} \mathrm{O}$. The appropriate number of PEG molecules for the different reactions (from 10 to 0.08 molecules $/ \mathrm{nm}^{2}$ ) was then added into 8 microcentrifuge tubes. $1 \mathrm{ml}$ of the particles was then added on top of the PEG volume, and was mixed immediately by vortexing. This was incubated 30 minutes at room temperature, then centrifuged at $8000 \mathrm{x} \mathrm{g}$ for 5 minutes to pellet the particles. $950 \mathrm{ul}$ of supernantant was removed, and the particles were resuspended in the $\sim 50$ ul remaining volume. 20 ul was then removed and loaded onto a $0.5 \%$ agarose gel, and separated by electrophoresis at 25 volts for 15 minutes then 100 volts for 45 minutes. The remaining particles were resuspended in $1 \mathrm{ml}$ of $1 \mathrm{mM}$ phosphate buffer $\mathrm{pH} 7.0$, which was then used to measure hydrodynamic diameter and $\zeta$-potential (see below). 


\section{Particle Characterization}

The absorption spectra of synthesized particles were measured as 1:2 dilutions (stock: $\mathrm{H}_{2} \mathrm{O}$ ) using a UV-1601PC spectrophotometer (Shimadzu). TEM images were obtained by adding 10 ul of a 1:5 (stock: $\mathrm{H}_{2} \mathrm{O}$ ) dilution of the particle solution onto carbon-coated copper grids, then imaged on a Hitachi HD2000 STEM (Hitachi Corp). Hydrodynamic diameter and $\zeta$-potential was measured on a Nano-ZS Zetasizer (Malvern) with particles resuspended in a $1 \mathrm{mM}$ phosphate buffer solution ( $\mathrm{pH} 7.0)$.

Particle diameters and length-width ratios ( $n=100$ per treatment) were determined in ImageJ using the "Particle Analyzer" function. Briefly, images were imported, and the scale determined by drawing a line across the image scale bar, then "Analyze, Set Scale" was chosen. Images were then cropped to exclude the caption. We then performed the following steps. "1. Image, Adjust, Threshold. 2. Process, Binary, Watershed. 3. Analyze, Set Measurements (including Ferret's Diameter and Fit Ellipse). 4. Analyze Particles, setting variables Size $=0$ 100000, Circularity=0-1, Show=Outline, Display Results (Yes) and Exclude on Edges (Yes). We then examined the resulting outline images to ensure proper analysis and excluded any unrepresentative outlines. 